\title{
Molecular AND Logic Gate Based on Electric Dichroism of a Photochromic Dihydroindolizine ${ }^{* *}$
}

Joakim Andréasson," Yuichi Terazono, Bo Albinsson, Thomas A. Moore, ${ }^{*}$ Ana L. Moore, ${ }^{*}$ and Devens Gust $^{*}$

Molecular switches that perform Boolean logic operations are scientifically interesting and may have technological potential. ${ }^{[1-4]}$ A variety of molecule-based logic switches (AND gates, OR gates, etc.) have been reported. ${ }^{[5-9]}$ In the vast majority, at least one of the gate inputs requires physical addition of

** We thank Prof. Bengt Nordén, Mr. Tore Eriksson, and M. Sc. Mattias Eng for helpful discussions and technical assistance. This work was supported by the Swedish Research Council (VR) and the U. S. National Science Foundation (CHE-0352599).

${ }^{*}$ Dr. J. Andréasson, Prof. Dr. B. Albinsson

Department of Chemistry and Bioscience

Chalmers University of Technology

SE-412 96 Göteborg, Sweden

FAX: (+46) 31-772-3858

E-mail: a-son@chalmers.se

Dr. Y. Terazono, Prof. Dr. T. A. Moore, Prof. Dr. A. L. Moore, Prof. Dr. D. Gust

Center for the Study of Early Events in Photosynthesis

Department of Chemistry and Biochemistry

Arizona State University

Tempe, AZ 85287, USA

FAX: (+1) 480-965-2747

E-mail: gust@asu.edu 
a chemical species. We have reported several molecular switches that employ photochromes, and function with only optical or thermal inputs. ${ }^{[9-12]}$ Orientation of molecules by electric fields can lead to optically-detectable electric linear dichroic phenomena. ${ }^{[13]}$ The ability to control solution absorbance using either photochromism or electric fields suggests that molecular logic gates switched by these two inputs might be realizable. Here, we report a molecular AND gate based on photochromic dihydroindolizine 1 (Figure 1) whose two inputs are light at $366 \mathrm{~nm}$ and an electric field, and whose output is the electric linear dichroism (ELD) of $\mathbf{1}$

Dihydroindolizine ${ }^{[14]}$ photochrome 1 exists in two isomeric forms. The closed, thermally stable spiro form 1c does not absorb light significantly in the visible (Figure 2); its longest-wavelength absorption band is at $415 \mathrm{~nm}$. Irradiation of 1c with ultraviolet light causes isomerization to the open, zwitterionic form 1o, which absorbs in the visible $\left(\lambda_{\max }=585 \mathrm{~nm}\right)$. Heat or visible light isomerize 1o back to 1c. At $20.5^{\circ} \mathrm{C}$, irradiation of a 2-methyltetrahydrofuran solution of $1 \mathrm{c}$ at $366 \mathrm{~nm}\left(\sim 1.5 \mathrm{~mW} / \mathrm{cm}^{2}\right)$ converts it to a photostationary state greatly enriched in 10 with a time constant of $10.6 \mathrm{~s}$. In the dark, this sample thermally isomerizes slowly back to $\mathbf{1 c}(\tau=580 \mathrm{~min})$. Irradiation of a sample in the $\mathbf{1 0}$ state with visible light $\left(580 \mathrm{~nm}<\lambda<900 \mathrm{~nm}, \sim 50 \mathrm{~mW} / \mathrm{cm}^{2}\right)$ leads to photoisomerization back to $1 \mathrm{c}(\tau=9.8$ $\min )$.

Electric linear dichroism (ELD) is the change in absorbance of plane-polarized light by a substance due to an applied electric field. Isotropically oriented molecules in an isotropic solvent have the same absorbance for plane-polarized light with any polarization relative to an external coordinate system. In the presence of an electric field, a molecule with a dipole moment, such as 1o, will partially align, orienting the transition moment for absorption, and anisotropic behavior results. The magnitude of the absorbance change observed upon application of a field depends upon, among other things, the dipole moment and the isotropic extinction coefficient. Molecule 10 is relatively polar, which favors alignment with an external field. It has an absorption maximum at $585 \mathrm{~nm}$, and could exhibit a 
significant ELD effect. Isomer 1c does not absorb in the visible spectral region, and is not expected to have a significant ELD response.

The ELD response for $\mathbf{1}$ was observed using apparatus detailed in the Experimental Section. The sample of 1 was placed in a cuvette containing two parallel flat plate metal electrodes separated by 1.6 mm. A monochromatic plane-polarized light beam was passed through the sample between the electrodes, with its plane of polarization parallel to the electric field generated by application of a voltage across the electrodes. The intensity of the transmitted light was monitored with a photomultiplier tube. To observe the ELD response, a voltage was imposed across the electrodes. A 1.5 $\mathrm{kV}$ AC electric field $(50 \mathrm{~Hz}, 940 \mathrm{kV} / \mathrm{m})$ was used, and the light intensity change induced by the field was measured using lock-in detection. An AC signal from the lock-in amplifier reflects a change in absorption of the sample due to the field (ELD response).

Figure 3 shows the ELD response (decrease in the intensity of transmitted light) for a sample of $\mathbf{1}$ that has been irradiated at $366 \mathrm{~nm}$, converting it to a photostationary state consisting mainly of $\mathbf{1 0}$. The large band at $\sim 583 \mathrm{~nm}$ is associated with the absorption of $\mathbf{1 0}$ (Figure 2). At $583 \mathrm{~nm}$ the signal-to-noise ratio is very high, and the ELD easily observable. The ELD response and the absorption spectra are not expected to match exactly, as the ELD signal has not been corrected for the (wavelength dependent) incident intensity and converted to logarithmic form. However, the lamp output as a function of wavelength is reasonably constant in this spectral region.

A similar experiment carried out with the closed form 1c (obtained by irradiation with red light) yields a small electric field response at the $\sim 416 \mathrm{~nm}$ absorption maximum (Figure 3 ). The response of 1c is much smaller than that of $\mathbf{1 0}$ due to differences in dipole moment, extinction coefficient and orientation of the transition dipole. The small response in regions where $\mathbf{1}$ does not absorb is ascribed to solvent, and it was observed even in the absence of molecule 1. Figure 3 also shows the ELD response for $\mathbf{1 0}$ and $\mathbf{1 c}$ in the absence of the electric field. The response is essentially zero in both cases. 
A Boolean AND logic gate requires two inputs, $A$ and $B$, and an output. In the initial state with no inputs, the gate does not generate an output. No output is produced when either input $A$ or input $B$ is switched on. However, if both inputs are on, the gate produces an output. Based on the ELD effects noted above, a solution of 1c can function as a molecule-based AND gate. Inputs $A$ and $B$ are UV irradiation at $366 \mathrm{~nm}$ and application of an electric field, as discussed above. The output of the gate is the ELD signal, monitored at $583 \mathrm{~nm}$.

In the initial state of the AND gate, the molecule is set in the thermally stable form 1c. In the absence of an electric field, this solution does not generate an ELD response, and the output of the gate is off (Figure 4). If this solution is irradiated at $366 \mathrm{~nm}$ (input $A$ on), a photostationary state containing mainly 10 is prepared. However, in the absence of an electric field (input $B$ off), no ELD response is observed, and the gate remains off. If, on the other hand, the original solution of 1c is subjected to the $50 \mathrm{~Hz}$ electric field (input $B$ on), only a small ELD response due to solvent is obtained. Given a suitable choice of threshold level, the gate remains off. Alternatively, when the solution of 1c is subjected to both $366 \mathrm{~nm}$ irradiation and the electric field, photoisomerization to 10 occurs, and 10 gives a strong ELD response at $583 \mathrm{~nm}$. The gate is switched on. Thus, the conditions for the AND function are met. After applying any combination of inputs, the gate may be reset to 1c by irradiation with red light.

Although the purpose of this research has been to investigate a new approach to molecular gates, rather than to make practical devices, the stability of $\mathbf{1}$ is important for any future applications. Figure 5a shows the ELD response of 10 during application of 10 on-off cycles of the electric field. There is no degradation of the signal. Figure $5 \mathrm{~b}$ reports the absorption spectra between 500 and $700 \mathrm{~nm}$ (absorption due to 1o) after each of ten 40-s exposures to $366 \mathrm{~nm}$ light alternated with nine 40-min exposures to red light. Only a small amount of photodegradation has occurred after these cycles. The sample was degassed with 6 freeze-pump-thaw cycles to a final pressure of $\sim 1 \times 10^{-4}$ torr prior to the experiment to reduce photodegradation. ${ }^{[15]}$ 
This molecular AND gate does not require the addition of chemicals or translational diffusion of molecules. The times required for the photoisomerizations involved in one input and in the resetting operation are determined by the light flux applied. The light intensities used here were chosen for convenience, rather than switching speed. The photoisomerization reactions themselves occur in ns or less. The time required for readout is ultimately limited by the time required to sufficiently align the molecular dipoles. This will be on the order of rotational diffusion times. If AC detection is employed the frequency of the applied AC field and time constant of the cell circuit will determine the time required for detection.

The UV photonic input to the AND gate may be applied at any time prior to readout, and the system will "remember" that this input was turned on due to the photoisomerization. The gate will persist in the 10 state until eventual thermal reversion. However, the second input, the AC high voltage, must be on during readout, or no ELD will be detected. This behavior differs from that of photonic gates where both inputs are photoisomerization reactions, and the final state of the gate may be read out at any convenient time after the inputs have been applied and turned off. ${ }^{[9,12]}$

In summary, a solution of photochrome 1 functions as a molecule-based AND Boolean logic gate, with inputs of 366-nm light and an AC electric field. The output is the electric linear dichroism response. Resetting the gate to the initial state is accomplished by irradiation with red light. The signalto-noise ratio is more than ample for observation of gate switching, and the sample can be cycled many times before photodegradation interferes. The apparatus is relatively simple, requiring only UV, visible, and plane-polarized light, a light-sensitive detector, an AC voltage source, and, for high sensitivity, lock-in detection.

\section{Experimental Section}

Dihydroindolizine 1 was synthesized as reported. ${ }^{[14]}$ The solvent for all measurements was freshly distilled 2-methyltetrahydrofuran. Photoisomerizations of $\mathbf{1}$ were achieved using a UVP UV lamp 
UVGL-25 $\left(366 \mathrm{~nm}, \approx 1.5 \mathrm{~mW} / \mathrm{cm}^{2}\right)$ or a $1000 \mathrm{~W}$ Xe/Hg-lamp at $450 \mathrm{~W}$. The Xe/Hg-lamp light was filtered by two hot mirrors (each having $A=1.8$ at $900 \mathrm{~nm}$ ) to reduce the IR intensity, and a long pass filter $(>580) \mathrm{nm}$. The power density at the sample was $\approx 50 \mathrm{~mW} / \mathrm{cm}^{2}$.

The electric linear dichroism (ELD) measurements were performed using a JASCO J-500 A CD spectropolarimeter with the photoelastic modulator disconnected, so that the incident light was linearly polarized parallel to the applied electric field. The $10 \times 10 \mathrm{~mm}$ silica cuvette was equipped with two planar stainless steel electrodes, $9.5 \mathrm{~mm}$ by $22 \mathrm{~mm}$, connected to a high voltage power supply at $50 \mathrm{~Hz}$ AC. The gap between the electrodes was $1.6 \mathrm{~mm}$, and the voltage was $1.5 \mathrm{kV}$, giving a maximal field strength of $940 \mathrm{kV} / \mathrm{m}$. The light passing between the electrodes was detected using a photomultiplier tube (PMT) connected to a lock-in amplifier (LIA) (EG\&G Model 124 A) equipped with a differential preamplifier (EG\&G Princeton Applied Research Model 116). The LIA receives a $50 \mathrm{~Hz}$ reference signal from the high voltage power supply. The LIA detects only the AC component of the PMT response in phase with the high voltage. The output of the LIA, representing the raw ELD response, is shown in the Figures.

\section{Reference List}

[1] A. Aviram, J. Am. Chem. Soc. 1988, 110, 5687-5692.

[2] V. Balzani, A. Credi, M. Venturi, Chem. Phys. Chem. 2003, 3, 49-59.

[3] A. P. de Silva, D. B. Fox, T. S. Moody, S. M. Weir, Pure Appl. Chem. 2001, 73, 503-511.

[4] P. Ball, Nature 2000, 406, 118-120.

[5] D. Kuciauskas, P. A. Liddell, A. L. Moore, T. A. Moore, D. Gust, J. Am. Chem. Soc. 1998, 120, 10880-10886.

[6] F. M. Raymo, S. Giordani, J. Am. Chem. Soc. 2002, 124, 2004-2007. 
[7] M. N. Stojanovic, T. E. Mitchell, D. Stefanovic, J. Am. Chem. Soc. 2002, 124, 3555-3561.

[8] X. Guo, D. Zhang, G. Zhang, D. Zhu, J. Phys. Chem. B 2004, 108, 11942-11945.

[9] J. Andréasson, G. Kodis, Y. Terazono, P. A. Liddell, S. Bandyopadhyay, R. H. Mitchell, T. A. Moore, A. L. Moore, D. Gust, J. Am. Chem. Soc. 2004, 126, 15926-15927.

[10] P. A. Liddell, G. Kodis, J. Andréasson, L. de la Garza, S. Bandyopadhyay, R. H. Mitchell, T. A. Moore, A. L. Moore, D. Gust, J. Am. Chem. Soc. 2004, 126, 4803-4811.

[11] S. D. Straight, J. Andréasson, G. Kodis, A. L. Moore, T. A. Moore, D. Gust, J. Am. Chem. Soc. 2005, 127, 2717-2724.

[12] S. D. Straight, J. Andréasson, G. Kodis, S. Bandyopadhyay, R. H. Mitchell, T. A. Moore, A. L. Moore, D. Gust, J. Am. Chem. Soc. 2005, 127, 9403-9409.

[13] N. Keitaro, Y. Atassi, J. A. Delaire, MCLC S\&T, Section B: Nonlinear Optics 1996, 15, 351358.

[14] H. Dürr, in Organic Photochromic and Thermochromic Compounds, Vol. 1, (Eds.: J. C. Crano, R. J. Guglielmetti), Plenum Press, New York 1999, pp. 223-266.

[15] H. Dürr, H. Gross, Agfa-Gevaert N. V. , Neth. Research Disclosure 1981, 205, 188-190. 


\section{Figure Captions}

Figure 1. Structures of the closed (1c) and open (10) isomers of the dihydroindolizine. Thermally stable 1c may be photoisomerized to 10 using $366 \mathrm{~nm}$ UV light and 10 may be isomerized to 1c using visible light or heat.

Figure 2. Absorption spectra of 2-methyltetrahydrofuran solutions of the open, 10 ( $\longrightarrow$ ), and closed, 1c $(----)$, forms of the photochrome.

Figure 3. ELD response in arbitrary units of a solution of $\mathbf{1}$ in 2-methyltetrahydrofuran: $(\longrightarrow)$ following 366-nm irradiation and with application of electric field, ( $\square \quad \square \quad \square$ ) following red light irradiation and with application of electric field, $(\bullet \bullet \bullet)$ following 366-nm irradiation and in absence of electric field, and ( $\square \bullet \bullet \square$ ) following red light irradiation and in the absence of electric field. Note that the two data sets in the absence of the electric field overlap.

Figure 4. ELD response (arbitrary units) at $583 \mathrm{~nm}$ of $\mathbf{1}$ in 2-methyltetrahydrofuran following combinations of light (UV) and electric field (HV) inputs for the AND logic gate (abscissa). Prior to the indicated inputs, the sample was in the 1c state. The signal-to-noise ratio (variation of the ELD signal with time during a 4-s collection period, 20 data points) is shown by the small height variations within each bar.

Figure 5. Cycling of AND gate switching operations. (a) The ELD signal at $583 \mathrm{~nm}$ from 1 in 2methyltetrahydrofuran, measured for ca. $15 \mathrm{~s}$ after each switching operation, as a function of cycling of the applied voltage (HV) between $1.5 \mathrm{kV}$ (HV on) and $0 \mathrm{~V}(\mathrm{HV}$ off). (b) Sets of absorption spectra of 1, each recorded between 500 and $700 \mathrm{~nm}$ where the zwitterionic isomer 10 absorbs: after UV irradiation (ם), and after red light irradiation $(\square)$, as described in the text. 
Figure 1

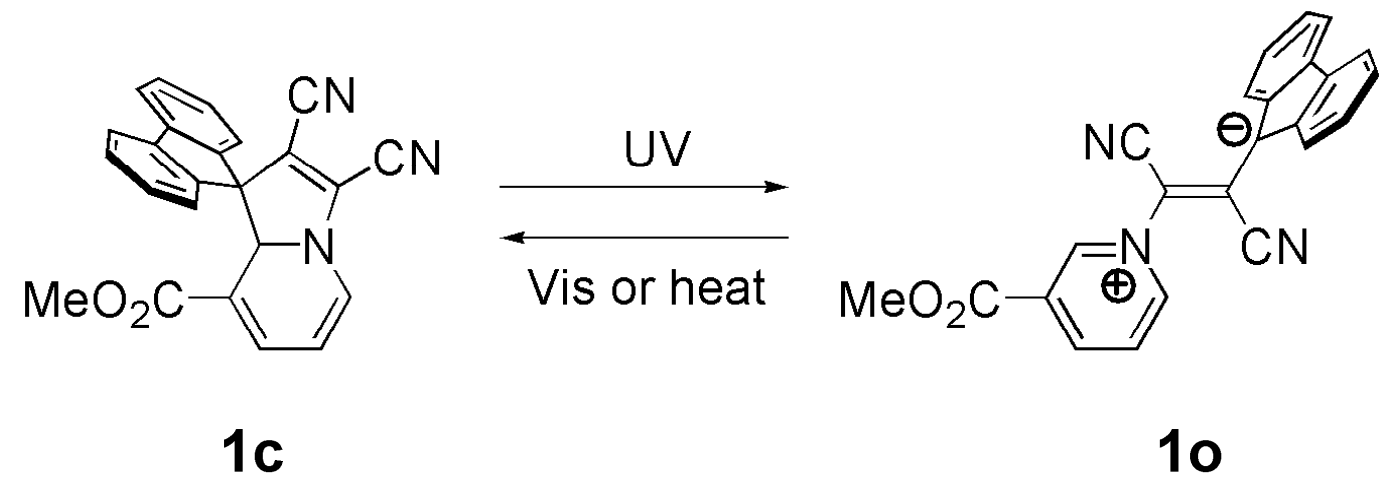


Figure 2

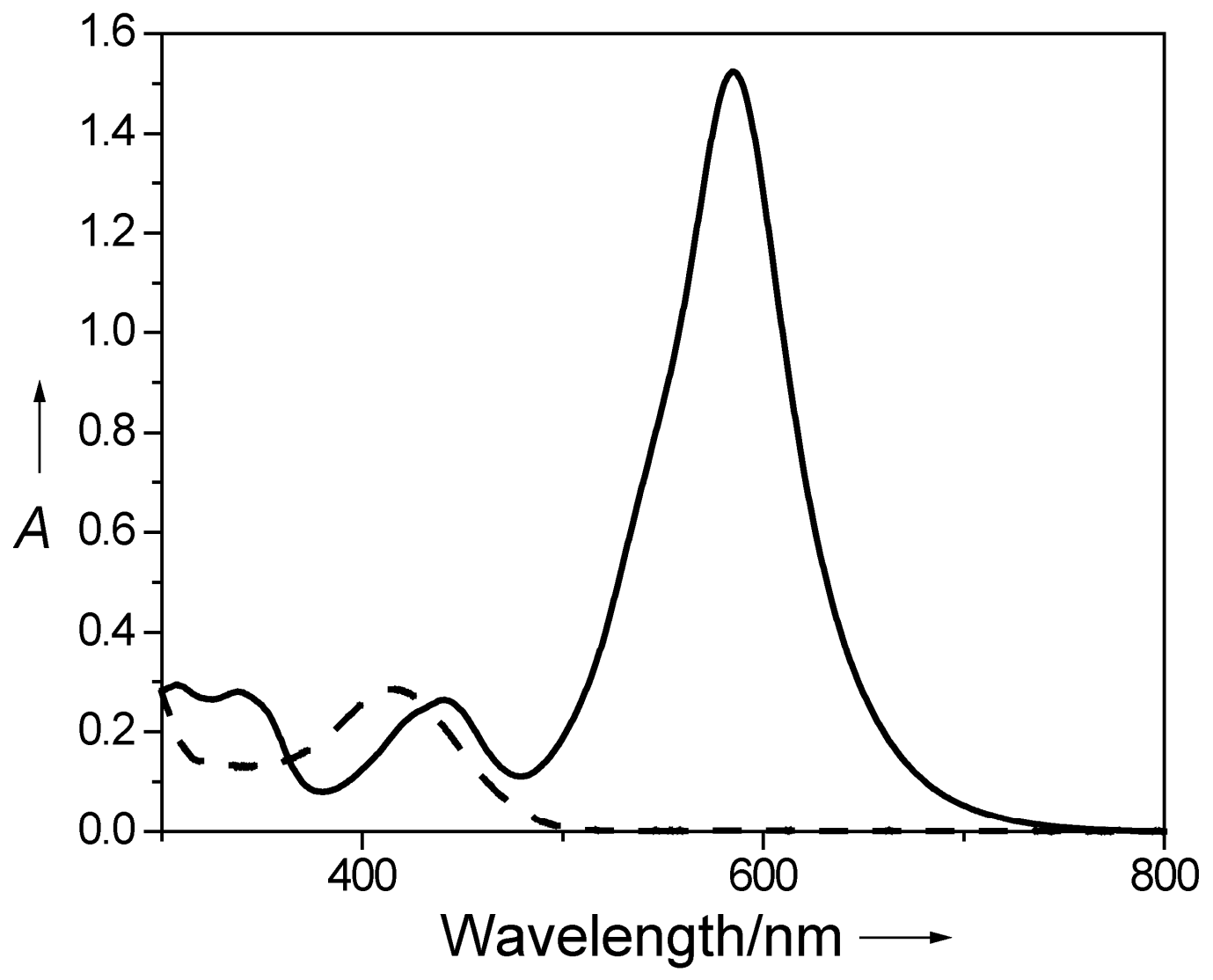


Figure 3

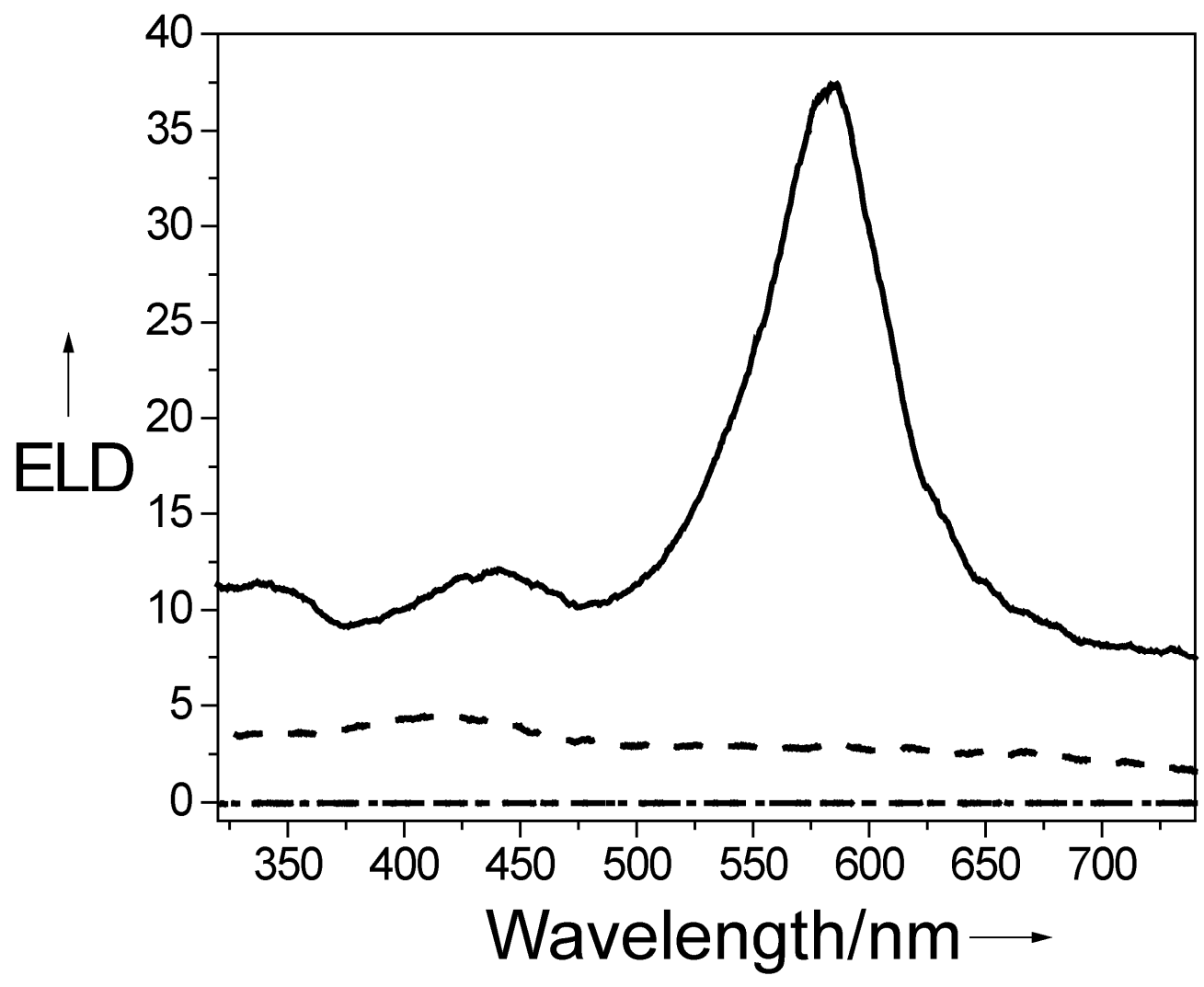


Figure 4

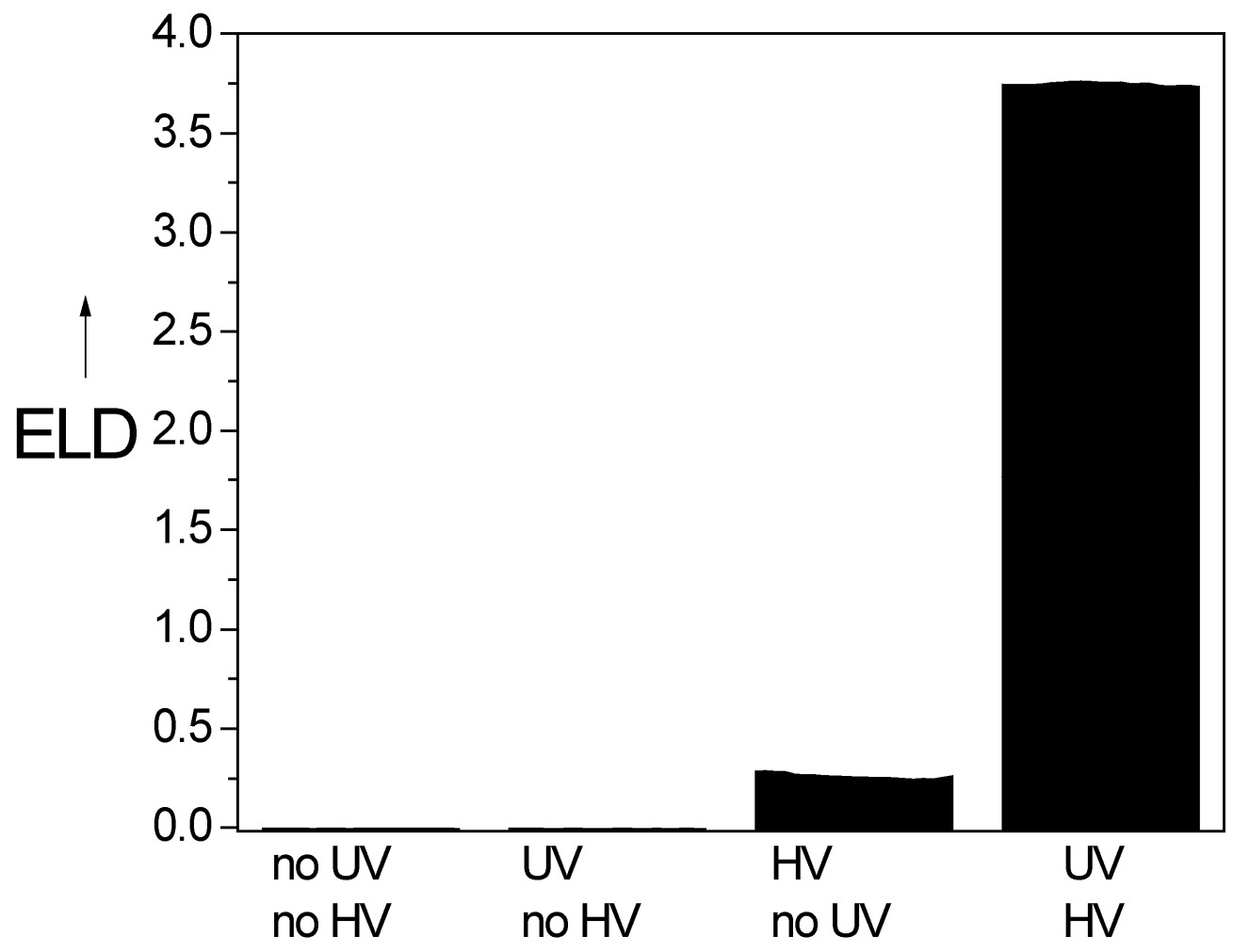


Figure 5
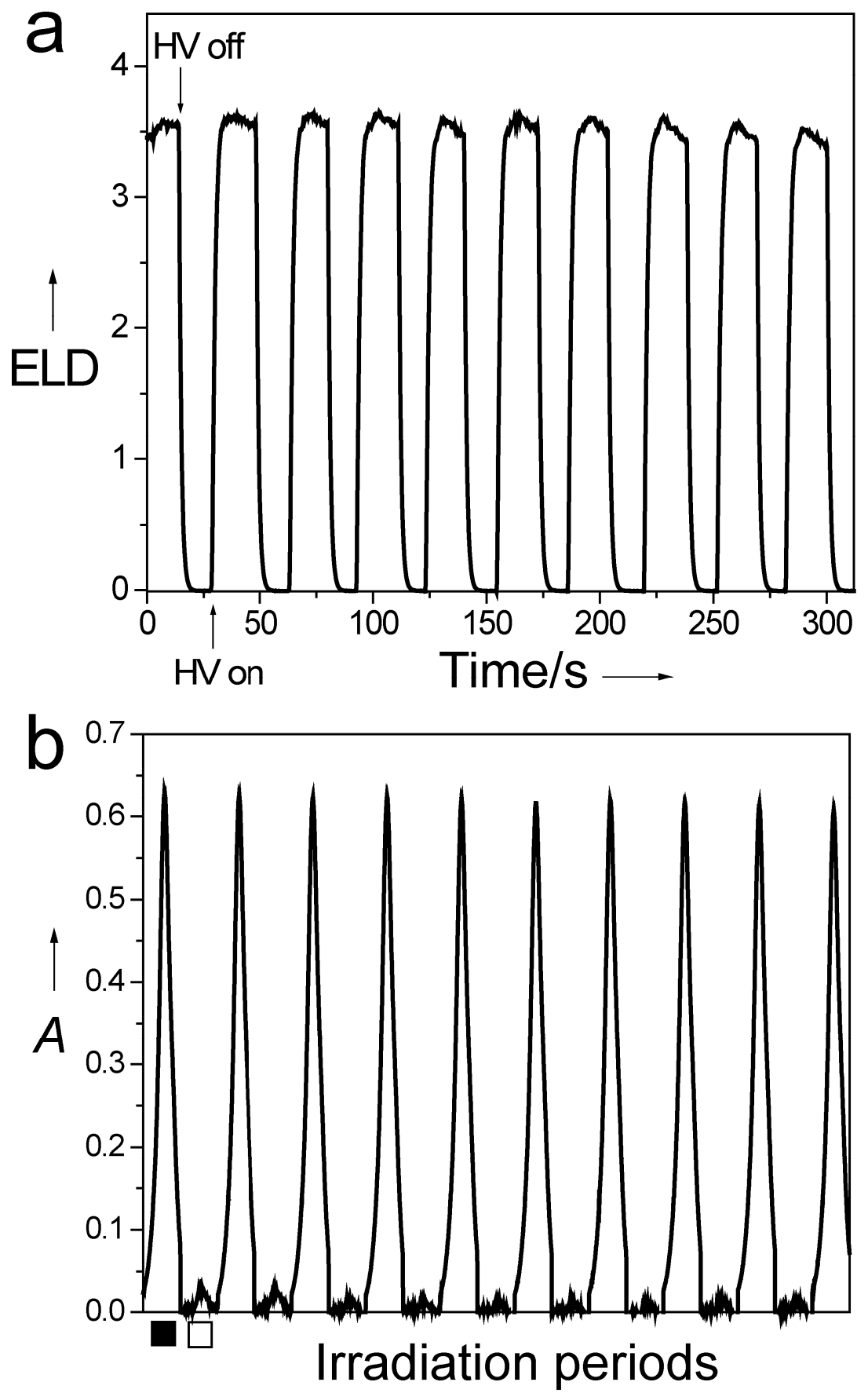


\section{Text for Table of Contents}

A photochromic molecular switch acts as an AND Boolean logic gate. The two inputs are UV light, which causes photoisomerization of a dihydroindolizine, and an electric field, which aligns the visibleabsorbing zwitterionic isomer in solution. The output of the gate is the electric linear dichroism response of the visible-absorbing isomer, which is only observed when both of the above inputs have been switched on. The gate is reset using visible light.

\section{Keywords}

Photochromism

Molecular devices

Electric field

Polarized spectroscopy

Electric linear dichroism 
TOC Graphic

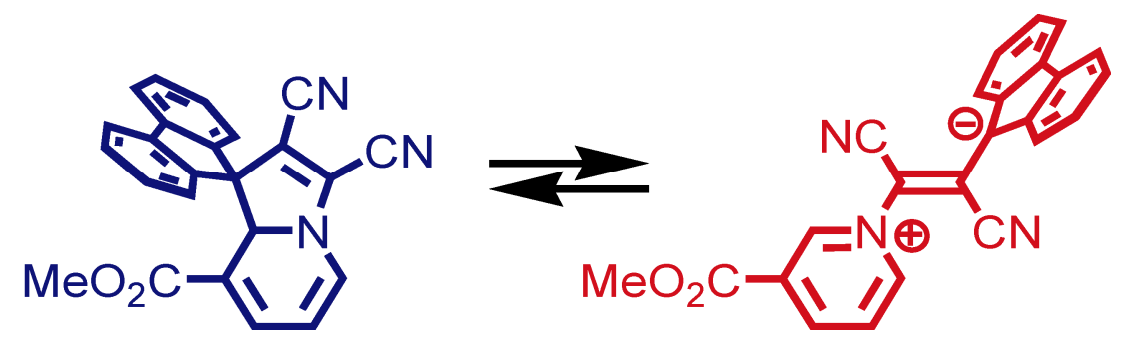

